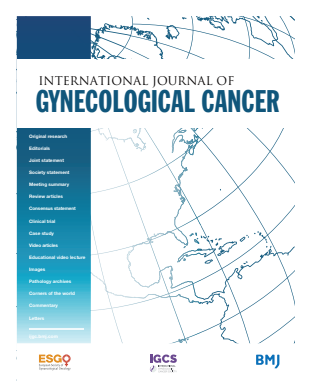

\title{
Adjuvant chemoradiation associated with improved outcomes in patients with microsatellite instability-high advanced endometrial carcinoma
}

Jennifer McEachron (D) , ${ }^{1}$ Nancy Zhou, ${ }^{1}$ Christina Spencer, ${ }^{2}$ Carolyn Chatterton, ${ }^{2}$ Lisa Shanahan, ${ }^{2}$ Julie Katz, ${ }^{1}$ Saskia Naegele, ${ }^{1}$ Pankaj K Singhal, ${ }^{2}$ Yi-Chun Lee ${ }^{1}$

${ }^{1}$ Gynecologic Oncology, SUNY Downstate Medical Center Health Sciences University, Brooklyn, New York, USA ${ }^{2}$ Gynecologic Oncology, Good Samaritan Hospital Medical Center, West Islip, New York, USA

Correspondence to Dr Jennifer McEachron, Gynecologic Oncology, SUNY Downstate Medical Center - Health Sciences University, Brooklyn, NY 11203, USA; jennifer.mceachron@ downstate.edu

Received 9 June 2020 Revised 24 July 2020 Accepted 28 July 2020 Published Online First 17 August 2020

\section{Check for updates}

(C) IGCS and ESGO 2021. No commercial re-use. See rights and permissions. Published by BMJ.

To cite: McEachron J, Zhou N, Spencer C, et al. Int J Gynecol Cancer 2021;31:203-208.

\section{HIGHLIGHTS}

- There is no consensus on the optimal adjuvant treatment of advanced endometrial cancer.

- Evidence suggests microsatellite instability (MSI)-high tumors derive significant benefit from radiotherapy.

- Chemoradiation was associated with improved survival versus chemotherapy-alone in MSI-high tumors.

\section{OBJECTIVES}

Evidence suggests that patients with microsatellite instability (MSI)-high endometrial carcinoma derive greater benefit from radiation therapy than their microsatellite-stable counterparts. We sought to evaluate the outcomes of patients with MSI-high advanced endometrial cancer treated with a combination of chemotherapy and radiation (chemoradiation) versus chemotherapy-alone to determine if there is a survival benefit associated with a particular adjuvant therapy regimen.

\section{METHODS}

A multicenter retrospective analysis of patients with stage III/IV, MSI-high endometrial carcinoma was conducted from January 2000 to December 2018. Inclusion criteria were primary surgical management, defined as hysterectomy with/without salpingoophorectomy, comprehensive surgical staging and/or tumor debulking, followed by adjuvant chemotherapy or chemoradiation. MSI status was determined by immunohistochemistry and/or next-generation sequencing. Differences in the frequencies of histology, stage, cytoreduction status, treatment delays, and sites of disease recurrence were identified using Pearson's chi-square test. Progression-free and overall survival were calculated using Kaplan-Meier estimates.

\section{RESULTS}

Final analysis included 37 patients; 20 (54\%) received chemoradiation and $17(46 \%)$ received chemotherapy-alone. The mean age was 62 (range $51-81)$ years. Histology included $48.6 \% \quad(n=18)$ endometrioid, $40.5 \%(n=15)$ serous, and $10.7 \%$ $(n=4)$ clear cell tumors. There was no difference in the frequency of histologic subtypes $(p=0.83)$, stage $(p=0.12)$, cytoreduction status $(p=0.45)$, treatment delays $(p=0.63)$, or location of recurrence $(p=0.89)$ between cohorts. There was a trend towards greater pelvic recurrence in the chemotherapy-alone cohort (36\% vs $29 \%$; $p=0.16$ ). The most frequent location of disease recurrence was the abdomen. The median progression-free survival favored chemoradiation (24 months) versus chemotherapy-alone (17 months) $(p=0.04)$. There was a trend towards improved overall survival in patients receiving chemoradiation (35 months) versus chemotherapy-alone (22 months) $(p=0.09)$. Chemoradiation demonstrated superiority over chemotherapy-alone in terms of 2-year progression-free $(40.0 \%$ vs $29.5 \%$, respectively; $\mathrm{p}=0.04)$ and 2-year overall survival $(73.7 \%$ vs $52.9 \%$, respectively; $p=0.09$ ).

\section{CONCLUSIONS}

Post-operative adjuvant therapy with a combination of chemotherapy and radiation was associated with improved survival compared with chemotherapyalone in patients with MSI-high advanced endometrial carcinoma.

\section{INTRODUCTION}

Endometrial cancer represents the most common gynecologic malignancy in the United States, affecting 61880 patients in 2019. ${ }^{1}$ Microsatellite instability (MSI) is a result of loss of function of mismatch repair (MMR) genes, and is identified in $20 \%-40 \%$ of all endometrial cancers. Loss of function of MMR genes leads to accumulation of single 


\section{Original research}

base-pair mismatches, as well as small insertions and deletions in tandem repeats, ultimately culminating in faulty double-stranded DNA repair and failure of apoptotic pathways. ${ }^{2}{ }^{3}$ Recently, The Cancer Genome Atlas classified endometrial cancer into four prognostic subgroups based on molecular tumor profiling. MSI was designated as one of these four categories based on its distinct molecular profile characterized by very high mutation frequency. Notably, germline mutations in one or more of the MMR proteins are only identified in $3 \%-5 \%$ of all endometrial cancers. ${ }^{4}$ The majority of cases of $\mathrm{MSI}$ are due to sporadic mutations, most commonly hypermethylation of MLH1. ${ }^{5}$

MSI can be assessed by immunohistochemical loss of one or more of the MMR proteins, including MLH1, MSH2, MSH6, and/or PMS2, polymerase chain reaction (PCR) or next-generation DNA sequencing. ${ }^{6}$ Next-generation sequencing offers the advantage of allowing hundreds of genes to be sequenced simultaneously for each patient, which allows for a far greater number of MSI markers to be examined while additionally assessing other gene loci for actionable mutations. Compared with PCR, next-generation sequencing has been proven to be at least as accurate in the detection of MSI on a molecular level. Additionally, it is more costeffective, by eliminating the need for separate conventional PCR testing. ${ }^{7}$

The majority of endometrial cancers are diagnosed at at early stage with an overall good prognosis. However, approximately onethird of patients are diagnosed with advanced disease. ${ }^{89}$ Despite excellent outcomes in early-stage disease, the 5-year survival declines dramatically in advanced stages, with a 5 -year survival of $57 \%-69 \%$ in stage III disease and a dismal $0 \%-18 \%$ survival reported in stage IV disease. ${ }^{10-14}$ The relationship between MSI and clinical outcomes is not clearly defined. Multiple studies have reported improved survival in MSI-high tumors versus microsatellite-stable tumors receiving the same adjuvant therapy; ${ }^{15}$ however, others have observed no difference or worse survival in MSI-high tumors. ${ }^{61617}$

Randomized clinical trials have consistently demonstrated a survival benefit with the use of chemotherapy in advanced disease ${ }^{1011}$ however, the role of radiation therapy in this patient population is less clear. Several authors have reported that combination adjuvant therapy with both systemic chemotherapy and radiotherapy produces superior clinical outcomes compared with either modality alone. ${ }^{18-22}$ There is, however, a lack of prospective data demonstrating improvements in survival with the addition of radiation. The recently published GOG 258 revealed a decrease in locoregional recurrence with the combination of chemotherapy and radiotherapy at the expense of greater distant failure compared with chemotherapy-alone and failed to demonstrate a survival benefit with combination adjuvant therapy. ${ }^{23}$ Notably, recent evidence suggests patients with MSI-high tumors derive greater benefit from radiation therapy than their microsatellite-stable counterparts. ${ }^{62425}$ In the present study, we evaluated outcomes of MSI-high advanced endometrial cancer treated with a combination of chemotherapy and radiation therapy (chemoradiation) versus chemotherapy-alone to determine if there is a survival benefit associated with a particular adjuvant therapy regimen.

\section{METHODS}

A multicenter retrospective analysis of patients with stage III/IV, MSI-high endometrial cancer was conducted from January 2010 to December 2018. Internal review board approval was obtained at all participating institutions. Tumor registries were reviewed to identify all patients with advanced endometrial cancer who received primary surgical treatment, followed by adjuvant therapy with chemotherapy-alone or chemoradiation. Inclusion criteria were patients with MSI-high endometrial cancer who had undergone primary surgical management, consisting of hysterectomy with/ without bilateral salpingoophorectomy, comprehensive surgical staging and/or tumor debulking, followed by adjuvant chemotherapy or chemoradiation. Comprehensive surgical staging was defined as pelvic with/without para-aortic lymph node dissection with/without omentectomy. Tumor debulking was defined as removal of extrauterine gross tumor from the abdominopelvic cavity. Receipt of chemoradiation was defined as patients receiving chemotherapy and external beam radiotherapy with/without vaginal brachytherapy. Tumors were designated as MSI-high by the absence of MMR proteins on immunohistochemistry or MSI-high on nextgeneration sequencing. Exclusion criteria included microsatellitestable tumors, patients with incomplete surgical staging, patients receiving neoadjuvant chemotherapy and/or pre-operative radiotherapy, and patients receiving vaginal brachytherapy without external beam radiotherapy.

Clinical and demographic data were obtained from tumor registries, operative notes, pathology reports, and both inpatient and outpatient medical records. Data regarding date of diagnosis, surgical procedures, cytoreduction status, types of adjuvant therapy, date and site(s) of recurrence, chemotherapy regimen, number of chemotherapy cycles received, type of radiation therapy received, treatment delays, adverse events, and date of death were extracted. Optimal cytoreduction was defined as all residual tumor $\leq 1 \mathrm{~cm}$ in diameter at the completion of cytoreductive surgery. Both optimally and suboptimally cytoreduced patients were included in the final analysis. A one-way ANOVA test was used to compare the differences in mean age between treatment arms. Differences in the frequencies of histology, stage, and cytoreduction status were analyzed using Pearson's chi-square test. Progression-free survival was defined as time from initial surgery to time of first recurrence, disease progression, or death. Overall survival was defined as time from initial surgery to time of death. Progressionfree and overall survival rates were calculated using Kaplan-Meier estimates. Statistical significance was defined as $p<0.05$. Analysis was performed using SPSS version 25.0 (IBM, Armonk, NY, USA).

\section{RESULTS}

A total of 184 patients with advanced endometrial cancer undergoing primary surgical management followed by chemotherapy with/without radiotherapy were identified. Thirty-seven (20.1\%) patients were found to have MSI by next-generation sequencing or immunohistochemistry. Among them, 17 (46\%) received chemotherapy-alone and 20 (54\%) received chemoradiation. The mean age was 62 (range 51-81) years and the majority of patients in both treatment arms were African-American (76\%). Stage distribution included $67.6 \%(n=25)$ stage III and $32.4 \%(n=12)$ stage IV 
Table 1 Patients' clinical and pathologic characteristics

\begin{tabular}{|c|c|c|c|c|}
\hline Characteristic & All patients $(n=37)$ & $C(n=17)$ & CR $(n=20)$ & P value \\
\hline Age at surgery (years) (mean (range)) & $62(51-81)$ & $63(54-81)$ & $61(51-77)$ & 0.18 \\
\hline \multicolumn{5}{|l|}{ Race (n (\%)) } \\
\hline Caucasian & $10(27)$ & $4(24)$ & $6(30)$ & 0.66 \\
\hline African-American & $27(73)$ & $13(76)$ & $14(70)$ & \\
\hline \multicolumn{5}{|l|}{ FIGO stage (n (\%)) } \\
\hline IIIA & $1(3)$ & $0(0)$ & $1(5)$ & 0.12 \\
\hline IIIB & $3(8)$ & $1(6)$ & $2(10)$ & \\
\hline IIIC1 & $12(32)$ & $6(35)$ & $6(30)$ & \\
\hline IIIC2 & $9(25)$ & $4(24)$ & $5(25)$ & \\
\hline IVA & $0(0)$ & $0(0)$ & $0(0)$ & \\
\hline IVB & $12(32)$ & $6(35)$ & $6(30)$ & \\
\hline \multicolumn{5}{|l|}{ Cytoreduction status (n (\%)) } \\
\hline Optimal & $34(92)$ & $15(88)$ & 19 (95) & 0.45 \\
\hline Suboptimal & $3(8)$ & $2(12)$ & $1(5)$ & \\
\hline \multicolumn{5}{|l|}{ Histology (n (\%)) } \\
\hline Endometrioid & $18(49)$ & $9(53)$ & $9(45)$ & 0.83 \\
\hline Serous & $15(40)$ & $7(41)$ & $8(40)$ & \\
\hline Clear cell & $4(11)$ & $1(6)$ & $3(15)$ & \\
\hline
\end{tabular}

C, chemotherapy-alone; CR, chemoradiation; FIGO, International Federation of Obstetrics and Gynecology.

disease. Histologic subtypes included $48.6 \%(\mathrm{n}=18)$ endometrioid, $40.5 \%(n=15)$ serous, and $10.7 \%(n=4)$ clear cell tumors. Thirtyfour (92\%) patients underwent optimal cytoreduction. There was no difference in the distribution of age $(p=0.18)$, race $(p=0.66)$, stage $(p=0.12)$, frequency of histologic subtypes $(p=0.83)$, or cytoreduction status $(p=0.45)$ between treatment regimens (Table 1$)$.

Seventeen $(46.0 \%)$ patients were diagnosed as MSI-high by immunohistochemistry-alone, $13(35.1 \%)$ by molecular tumor profiling with next-generation sequencing-alone, and seven $(18.9 \%)$ by both modalities. Nine patients had both immunohistochemistry and molecular tumor profiles available for review. Of these patients, seven $(77.8 \%)$ were identified as MSI-high by both methods. The remaining two patients were designated as MSIhigh by next-generation sequencing only, but found to have intact MMR expression by immunohistochemistry. Of the patients who were MMR-deficient by immunohistochemistry, the most common molecular aberration was loss of expression of MLH1 (Table 2).

The majority of patients received platinum-based chemotherapy $(97.3 \%)$, most commonly carboplatin-paclitaxel $(81.0 \%)$. Other regimens included cisplatin-paclitaxel-doxorubicin (13.6\%), cisplatin-doxorubicin (2.7\%), and single-agent doxorubicin (2.7\%). The median number of cycles received was 6 (range 4-8). Of those patients receiving chemoradiation, the majority received external beam radiotherapy to the pelvis and extended-field to the para-aortic nodal area with/without vaginal brachytherapy $(55 \%)$. The remaining patients received pelvic external beam radiotherapy with/without vaginal brachytherapy $(45 \%)$. A total of $40 \%$ of patients received vaginal brachytherapy with external eam radiotherapy. Among the chemoradiation cohort, the sequence of therapy varied with $45 \%$ receiving chemotherapy followed by radiation, $15 \%$ receiving radiation followed by chemotherapy, and the
Table 2 Method used to diagnose microsatellite instability and specific molecular aberrations

\begin{tabular}{llll}
\hline Diagnostic method & C (n (\%)) & CR (n (\%)) & $\begin{array}{l}\text { Total (n)) } \\
\text { IHC }\end{array}$ \\
NGS & $6(47)$ & $9(45)$ & $17(46)$ \\
IHC + NGS & $3(18)$ & $7(35)$ & $13(35)$ \\
$\begin{array}{l}\text { Specific molecular } \\
\text { aberration }\end{array}$ & & & $7(19)$ \\
IHC & & & \\
$\quad$ MLH-1 & $3(17)$ & $5(25)$ & $8(22)$ \\
$\quad$ MSH-2 & $1(6)$ & $2(10)$ & $3(8)$ \\
$\quad$ MSH-6 & $1(6)$ & $2(10)$ & $3(8)$ \\
$\quad$ PMS-2 & $1(6)$ & $0(0)$ & $1(3)$ \\
$\quad$ MSH-2 + MSH-6 & $2(12)$ & $0(0)$ & $2(5)$ \\
NGS & & & \\
$\quad$ MSI-high & $6(35)$ & $7(35)$ & $13(35)$ \\
IHC + NGS & & & \\
$\quad$ MSI-high + MLH-1 & $2(12)$ & $2(10)$ & $4(11)$ \\
$\quad$ MSI-high + MSH-2 & $1(6)$ & $1(5)$ & $2(5)$ \\
MSI-high + MSH-6 & $0(0)$ & $1(5)$ & $1(3)$ \\
\hline
\end{tabular}

$\mathrm{C}$, chemotherapy-alone; $\mathrm{CR}$, chemoradiation; $\mathrm{IHC}$, immunohistochemistry; MSI, microsatellite instability; NGS, nextgeneration sequencing. 


\section{Original research}

Table 3 Recurrence site based on adjuvant therapy regimen

\begin{tabular}{lll}
\hline Parameter & $\begin{array}{l}\mathrm{C}(\mathbf{n}=\mathbf{1 7}) \\
(\mathbf{n}(\%))\end{array}$ & $\begin{array}{l}\mathrm{CR}(\mathbf{n}=\mathbf{2 0}) \\
(\mathbf{n}(\%))\end{array}$ \\
\hline Total recurrences & 15 & 13 \\
Total recurrence sites* & 22 & 17 \\
$\begin{array}{l}\text { Recurrence site } \\
\quad \text { Abdomen }\end{array}$ & & \\
$\quad$ Pelvis & $10(46)$ & $10(59)$ \\
Vagina & $8(36)$ & $5(29)$ \\
Extra-peritoneal & $2(9)$ & $0(0)$ \\
\hline
\end{tabular}

*Many patients recurred at more than one location concurrently; the numbers reflect each individual site of recurrence.

Consequently, the total number of recurrence sites exceeds the total number of recurrences in the population.

$\mathrm{C}$, chemotherapy-alone; $\mathrm{CR}$, chemoradiation.

remaining $40 \%$ receiving sandwich sequencing (chemotherapyradiation-chemotherapy). Two patients received radiosensitization with cisplatin during external beam radiotherapy. A total of $48.6 \%$ patients experienced a delay in treatment; $53 \%$ in the chemotherapy-alone cohort and $60 \%$ in the chemoradiation cohort. The most common reason for treatment delay in both treatment arms was neutropenia. A total of $18 \%$ of patients underwent dose chemotherapy dose reduction and $89 \%$ completed all prescribed chemotherapy cycles. There was no difference in the frequency of treatment delays between adjuvant therapy regimens $(p=0.63)$.

The most frequent location of disease recurrence was the abdomen ( $51.3 \%)$, followed by pelvis (33.3\%), extra-peritoneal sites $(10.3 \%)$, and vagina $(5.1 \%)$. The was no significant difference in the distribution of recurrence between cohorts $(p=0.89)$; however, there was a trend towards higher rate of pelvic failure in the

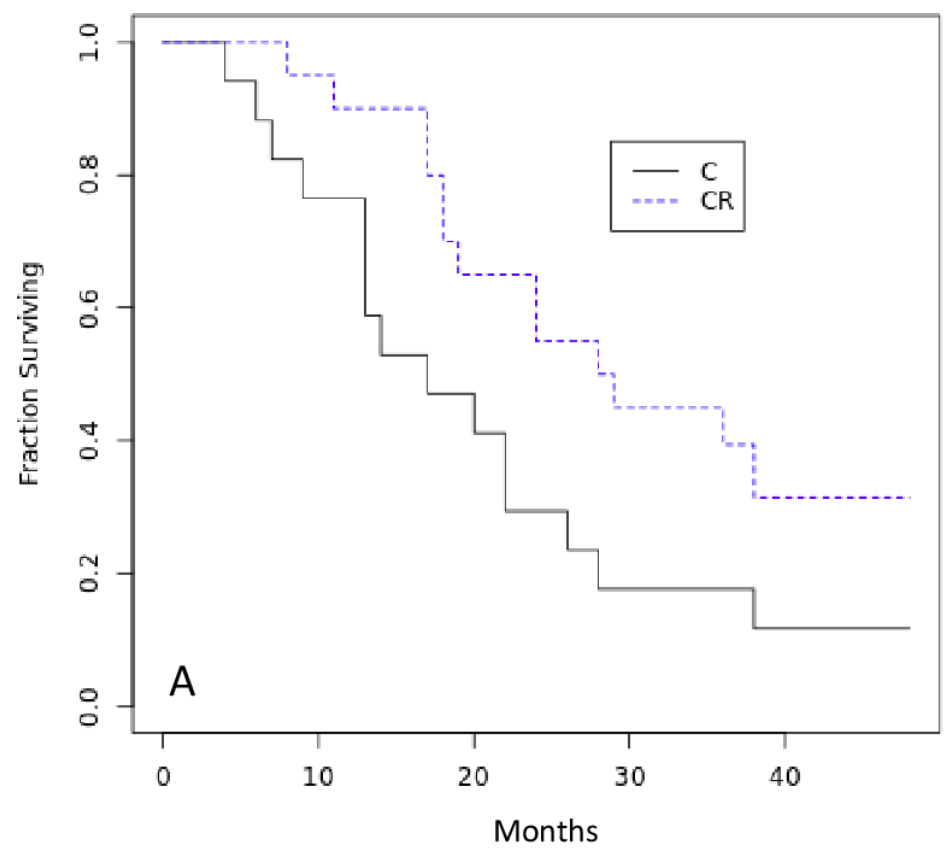

chemotherapy-alone cohort (36\%) compared with those receiving chemoradiation $(29 \%)(p=0.16)$. There was no vaginal cuff recurrence among the patients receiving chemoradiation (Table 3). There was a significant improvement in median progression-free survival in patients receiving chemoradiation compared with chemotherapyalone (24 vs 17 months, respectively; $p=0.04$ ). We observed a trend towards improved overall survival in patients receiving chemoradiation compared with those receiving chemotherapy-alone (35 vs 22 months; $p=0.09$ ). Chemoradiation also demonstrated superiority over chemotherapy-alone in terms of 2-year progression-free survival ( $40.0 \%$ vs $29.5 \%$, respectively) and overall survival $(73.7 \%$ vs $52.9 \%$, respectively) (Figure 1 ).

\section{DISCUSSION}

Current adjuvant treatment strategies for advanced endometrial cancer consist of systemic therapy with or without radiotherapy. ${ }^{9}$ A large body of retrospective literature demonstrates improved outcomes with the combination of chemoradiation; ${ }^{18-22}$ however, there are mixed prospective data supporting a survival benefit from the combination of chemoradiation. Two recent prospective randomized trials, PORTEC-3 and GOG 258, addressed the potential benefit of chemoradiation in locally advanced endometrial carcinoma. In PORTEC-3, patients were randomized to radiotherapy with concurrent cisplatin, followed by carboplatin-paclitaxel or to the standard arm, defined as external beam radiotherapy-alone. At a median of 72.6 months, final analysis demonstrated a significant failure-free and survival benefit with the combination of chemoradiation. ${ }^{26}$ Conversely, the recently published GOG 258 failed to demonstrate a recurrence-free survival benefit with the combination of chemoradiation versus the standard arm, consisting of chemotherapy-alone. The combination of chemoradiation was associated with a decrease in local recurrence at the expense of increased distant metastasis compared with chemotherapy-alone. ${ }^{23}$

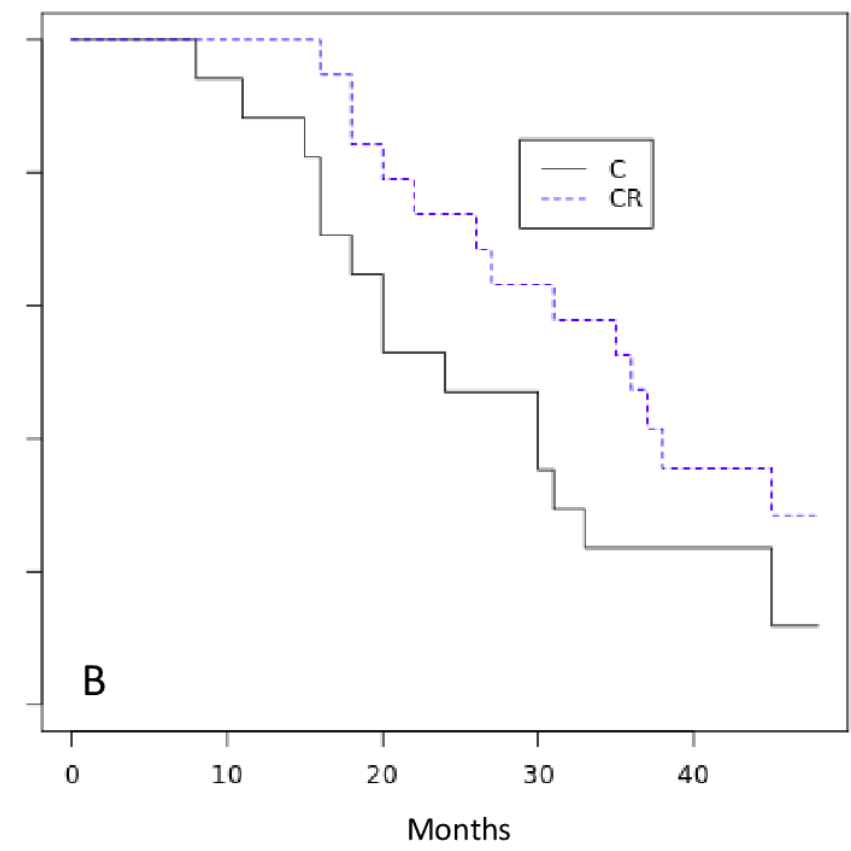

Months

Figure 1 Kaplan-Meier survival analysis by treatment group. (A) Progression-free survival analysis and (B) overall survival analysis. C, chemotherapy-alone; CR, chemoradiation. 
However, the chemoradiation arm received only four cycles of chemotherapy compared with the standard arm which received the traditional six cycles, leaving one to postulate whether the omission of two cycles from the experimental arm led to differences in outcomes. As a result of these two trials, there has been mixed utilization of chemoradiation, with some institutions adopting this strategy, while others await further prospective data.

However, the answer as regards which patients may derive the greatest benefit from the combination of chemoradiation may ultimately lie in the molecular characteristics of the tumor itself, rather than generalizing all advanced endometrial carcinomas to one treatment regimen. Evidence indicates that MMR proteins play a crucial role in repair of DNA damage following radiation therapy. Therefore, absence of these proteins leads to accumulation of DNA errors and enhances the effects of radiotherapy. Consistent with this theory, a recent study by Reijnen et al demonstrated improvement in disease-specific survival with the use of adjuvant radiation therapy versus observation in MMR-deficient early-stage endometrial carcinoma. ${ }^{24}$ Skikama et al analyzed a cohort of stage I-IV endometrial carcinoma and observed a trend towards improved survival in MMR-deficient tumors receiving adjuvant therapy versus MMR-proficient tumors undergoing the same regimens. Although the trend was observed across all stages, notably it was statistically significant in advanced-stage disease. ${ }^{15}$ Falling in line with these reports, the current study demonstrates a survival benefit from the addition of radiation therapy to chemotherapy in MSI-high patients. Conversely, a recent analysis of PORTEC3 based on molecular classification failed to demonstrate a benefit to chemoradiation over standard radiation therapy-alone in the MMR-deficient cohort. However, as the study did not include a chemotherapy-alone arm, conclusions cannot be drawn regarding the impact of radiation versus no radiation on MSI-high patients based on this trial. ${ }^{27}$

The rationale behind improved responsiveness to radiotherapy is based on the roles of MMR proteins in repairing radiation injury. MMR proteins are heavily involved in the repair of doublestranded DNA breaks, the most lethal form of radiation-induced DNA damage. ${ }^{28}$ Specifically, MLH1 and MSH2 recognize radiationinduced DNA damage and arrest the cell cycle at the G2/M phase transition. Additionally, loss of MLH1 decreases activation of nuclear factor kappa-beta, a protein that is normally activated after radiation-induced DNA damage and promotes cell survival by counteracting the tp53 apoptotic pathway. ${ }^{29} 30$ This illustrates the crucial roles MMR proteins play in DNA damage repair following radiation.

Current data demonstrate that MMR-deficient endometrial carcinomas exhibit a good response to programmed cell death protein-1 (PD-1) inhibition. A recent large prospective study of pembrolizumab in recurrent MMR-deficient tumors demonstrated an overall response rate of $54 \%$ with an additional $23 \%$ of patients experiencing stable disease. ${ }^{31}$ Further expanding these data, dostarlimab, another member of the PD-1 inhibitor family, demonstrated a $50 \%$ response rate in MSI-high recurrent endometrial cancer. ${ }^{32}$ The mechanism behind the efficacy of PD-L1 blockade in this population is due to the upregulation of the PD-1 pathway and the highly immunogenic nature of MMR-deficient tumors. ${ }^{33}$ Notably, recent evidence suggests that radiation enhances the efficacy of immune checkpoint blockade in vitro. ${ }^{34}$ In the light of these observations, multiple clinical trials are underway to investigate the role of radiation in combination with immune checkpoint blockers. ${ }^{35}{ }^{36}$ This adjuvant therapy combination theoretically could provide substantial benefit in MSI-high tumors.

A noteworthy finding of the current study is the discrepancy between MSI status noted in two patients. Of the entire cohort, nine patients underwent both immunohistochemistry and nextgeneration sequencing. Seven patients were confirmed MSI-high by both diagnostic methods. However, two patients were found to be MSI-high by next-generation sequencing and MMR-proficient by immunohistochemistry. This is explained by the fact that next-generation sequencing allows for a far greater number of microsatellite markers to be examined compared with immunohistochemistry and polymerase chain reaction-based testing. ${ }^{7}$ As we move further into the landscape of targeted therapy, treatment will be heavily dependent on accurate diagnosis of molecular aberrations, particularly MSI-high and MMR-deficient tumors. The two patients in our cohort could have been selected out as either MSIhigh or microsatellite-stable based on a single diagnostic modality, ultimately leading to expanded or limited treatment options.

The major limitations of the current study are its retrospective nature and small sample size. Despite these limitations, this study demonstrates a significant improvement in progression-free survival and a trend towards improved overall survival from the addition of radiation to chemotherapy in advanced-stage MSI-high endometrial carcinoma. We believe our findings are clinically significant, warranting future prospective evaluation and supporting the investigation of radiation-immunotherapy combinations in this patient population.

Contributors JMcE: conceptualization, methodology, formal analysis, writing original draft, visualization. NZ: data curation, writing - review and editing. CS: data curation. CC: data curation. LS: data curation. JK: data curation. SN: data curation. PKS: conceptualization, writing - review and editing. Y-CL: conceptualization, writing - review and editing, supervision, project administration.

Funding The authors have not declared a specific grant for this research from any funding agency in the public, commercial or not-for-profit sectors.

Competing interests None declared.

Patient consent for publication Not required.

Provenance and peer review Not commissioned; externally peer reviewed.

Data availability statement Data are available upon reasonable request. Deidentified data are available upon request.

ORCID iD

Jennifer McEachron http://orcid.org/0000-0002-3605-1163

\section{REFERENCES}

1 National Institutes of Health. National Cancer Institute Surveillance, Epidemiology, and End Results Program. Cancer stat facts: uterine cancer, 2019. Available: https://seer.cancer.gov/statfacts/html/corp. $\mathrm{html}$

2 Cancer Genome Atlas Research Network, Kandoth C, Schultz N, et al. Integrated genomic characterization of endometrial carcinoma. Nature 2013;497:67-73.

3 Backes FJ, Leon ME, Ivanov I, et al. Prospective evaluation of DNA mismatch repair protein expression in primary endometrial cancer. Gynecol Oncol 2009;114:486-90.

4 Bogani G, Tibiletti MG, Ricci MT, et al. Lynch syndrome-related non-endometrioid endometrial cancer: analysis of outcomes. Int $J$ Gynecol Cancer 2020;30:56-61.

5 Simkins SB, Bocker T, Swish EM, et al. MLH1 promoter methylation and gene silencing is the primary cause of microsatellite instability in sporadic endometrial cancer. Hum Mol Genet 1999;8:661-6.

6 Diaz-Padilla I, Romero N, Amir E, et al. Mismatch repair status and clinical outcomes in endometrial cancer: a systematic review and meta-analysis. Clin Rev Oncol Hem 2013;88:154-67. 


\section{Original research}

7 Salipante SJ, Scroggins SM, Hampel HL, et al. Microsatellite instability detection by next generation sequencing. Clin Chem 2014;60:1192-9.

8 Siegel RL, Miller KD, Jemal A. Cancer statistics, 2018. CA Cancer J Clin 2018;68:7-30.

9 National Comprehensive Cancer Network (NCCN) Guidelines. Uterine neoplasms. version 1.2020 2020.

10 Miller D, Filiaci V, Gleming G, et al. Randomized phase II noninferiority trial of first line chemotherapy for metastatic or recurrent endometrial carcinoma: a Gynecologic Oncology Group study. Gynecol Oncol 2012;125.

11 Fleming G, Brunetto V, Cella D, et al. Phase III trial of doxorubicin plus cisplatin with or without paclitaxel plus filgrastim in advanced endometrial carcinoma: a Gynecologic Oncology Group study. J Clin Oncol 2004;22:2159-66.

12 Greer BE, Hamberger AD. Treatment of intraperitoneal metastatic adenocarcinoma of the endometrium by whole abdomen moving strip technique and pelvic boost irradiation. Gynecol Oncol 1983;16:365-73.

13 Goff BA, Goodman A, Muntz HG, et al. Surgical stage IV endometrial carcinoma: a study of 47 cases. Gynecol Oncol 1994;52:237-40.

14 Bristow RE, Zerbe MJ, Rosenshein NB, et al. Stage IVb endometrial carcinoma: the role of cytoreductive surgery and determinants of survival. Gynecol Oncol 2000;78:85-91.

15 Shikama A, Minaguchi T, Matsumoto K, et al. Clinicopathologic implications of DNA mismatch repair status in endometrial carcinomas. Gynecol Oncol 2016;140:226-33.

16 McMeekin DS, Tritchler DL, Cohn DE, et al. Clinicopathologic significance of mismatch repair defects in endometrial cancer: an NRG Oncology/Gynecologic Oncology Group study. J Clin Oncol 2016;34:3026-68.

17 Zighelboim I, Goodfellow PJ, Goa F, et al. Microsatellite instability and epigenetic inactivation of MLH1 and outcomes of patients with endometrial carcinomas of the endometrioid type. J Clin Oncol 2007;25:2042-7.

18 Secord AA, Havrilesky LJ, Bae-Jump V, et al. The role of multimodality adjuvant chemotherapy and radiation in women with advanced stage endometrial cancer. Gynecol Oncol 2007;107:285-91.

19 Hoskins PJ, Swenerton KD, Pike JA, et al. Paclitaxel and carboplatin, alone or with irradiation in advanced or recurrent endometrial cancer: a phase II study. J Clin Oncol 2001;19:4048-53.

20 Albeesh R, Turgeon GA, Alfeirei J, et al. Adjuvant therapy in stage III endometrial cancer confined to the pelvis. Gynecol Oncol 2019;152:26-30.

21 Goodman CR, Hautoum S, Seagle BLL, et al. Association of chemotherapy and radiotherapy sequence with overall survival in locoregionally advanced endometrial cancer. Gynecol Oncol 2019.

22 Greven K, Winter K, Underhill K, et al. Final analysis of RTOG 9708: adjuvant postoperative irradiation combined with cisplatin/paclitaxel chemotherapy follow surgery for patients with high-risk endometrial cancer. Gynecol Oncol 2006;103:155-9.

23 Matei D, Filiaci V, Randall ME, et al. Adjuvant chemotherapy plus radiation for locally advanced endometrial cancer. $N$ Engl J Med 2019;380:2317-25.

24 Reijnen C, Kusters-Vandevelde HVN, Prinsen CF, et al. Mismatch repair deficiency as a predictive marker for response to adjuvant radiation therapy in endometrial cancer. Gynecol Oncol 2019;154:124-30.

25 Kim SR, Pina A, Albert A, et al. Dose MMR status in endometrial cancer influence response to adjuvant therapy? Gynecol Oncol 2018;151:76-81.

26 De Boer SM, Powell ME, Mileshkin L, et al. Adjuvant chemoradiotherapy versus radiotherapy alone in women with highrisk endometrial cancer (PORTEC-3): patterns of recurrence and post-hoc survival analysis of a randomized phase 3 trial. Lancet Oncol 2019;20:1273-85.

27 Creutzberg CL, Leon-Castillo A, de Boer SM, et al. Molecular classification of the PORTEC3 trial for high-risk endometrial cancer: impact on adjuvant therapy. ESMO Congress, LBA63 2019.

28 Chen J, Sadowski I. Identification of the mismatch repair genes pms2 and MLH1 as p53 target genes by using serial analysis of binding elements. Proc Natl Acad Sci U S A 2005;102:4813-8.

29 Franchitto A, Pichierri P, Piergentili R, et al. The mammalian mismatch repair protein MSH2 is required for correct MRE11 and RAD51 relocalization and for efficient cell cycle arrest induced by ionizing radiation in G2 phase. Oncogene 2003;22:2110-20.

30 Habraken Y, Jolois O, Piette J. Differential involvement of the hMRE11/hRAD50/NSB1 complex, BRCA1 MLH1 in NF-kB activation by captothecin and X-ray. Oncogene 2003;22:6090-9.

31 Le DT, Durham JN, Smith KN, et al. Mismatch repair deficiency predicts response of solid tumors to PD-1 blockade. Science 2017;357:409-13.

32 Oaknin A, Duska LR, Sullivan RJ, et al. Preliminary safety, efficacy and pharmacokinetic/pharmacodynamic characterization from GARNET, a phase I/II clinical trial of the anti-PD-1 monoclonal antibody, TSR-042, in patients with recurrent or advanced MSI-H and MSS endometrial cancer. Gynecol Oncol 2019.

33 Westdorp H, Fennemann FL, Weren RDA, et al. Opportunities for immunotherapy in microsatellite instable colorectal cancer. Cancer Immunol Immunother 2016;65:1249-59.

34 Vanpouille-Box C, Formenti SC, Demaria S. Toward precision radiotherapy for use with immune checkpoint blockers. Clin Cancer Res 2018;24:259-65.

35 Knag J, Demaria S, Formenti S. Current clinical trials testing the combination of immunotherapy with radiotherapy. J Immunother Cancer 2016.

36 Rodriguez-Ruiz ME, Rodriguez I, Leaman O, et al. Immune mechanisms mediating abscopal effects in radioimmunotherapy. Pharmacol Ther 2019;196:195-203. 